\section{Atlas of Genitourinary Oncological Imaging}

A.M. Bach and J. Zhang, Eds.

New York, NY: Springer, 2013, 343 pages, $\$ 189$

Most renal tumors arise from the renal parenchyma, with a much smaller number arising from the urothelium of the renal pelvis or the mesenchyma. Bladder cancer is the second most common genitourinary malignant disease, and its incidence increases with age. There has been a dramatic increase in the incidence of prostate cancer but also a shift toward earlier detection and improved survival. Testicular cancer is the most common malignancy in young men, and Wilms tumor and neuroblastoma make up the largest group of extracranial solid tumors of childhood.

This book is a comprehensive visual review of normal anatomy and findings of oncologic diseases in the genitourinary system, with extensive illustrations. Most of the contributors are part of the genitourinary disease management team at the Memorial SloanKettering Cancer Center, which is one of the premier cancer centers in the world, with cutting-edge technology and patient care. The 22 team members selected for their expertise are diagnostic radiologists, surgeons, and medical and radiation oncologists, and they are uniquely able to add to the care of the individual patient.

This book consists of 10 chapters, with the first six dealing with renal, upper urinary, bladder, prostate, adrenal, and testicular cancers. Chapter 7 discusses general chemotherapy imaging, and chapter 8 addresses genitourinary interventions. Chapter 9 outlines nuclear medicine imaging in genitourinary oncology, and the final chapter elaborates pediatric genitourinary tumor imaging. Each chapter includes introduction, diagnostic anatomy or presentation, imaging technique, pathology, staging of each cancer type, and expected as well as complicated imaging findings after treatment or management. Although the over 900 images are not glossy, abnormalities are well illustrated. The tables and index are helpful, and the references are updated.

I highly recommend this book to imaging and urology trainees seeking a review of basic information on anatomy and of imaging findings for genitourinary diseases. The book will also be of interest to practicing radiologists, nuclear physicians, and urologists looking for answers to challenging cases confronted in daily practice.

\section{E. Edmund Kim \\ University of California at Irvine \\ 101 The City Dr. S. \\ Orange, CA 92868 \\ E-mail: edmundek@uci.edu}

Published online Sep. 27, 2013.

DOI: $10.2967 /$ jnumed.113.132290

COPYRIGHT (C) 2013 by the Society of Nuclear Medicine and Molecular Imaging, Inc.
The Requisites: Nuclear Medicine

\author{
H.A. Ziessman, J.P. O’Malley, J.H. Thrall, and F.H. Fahey, Eds.
}

Philadelphia, PA: Saunders, 452 pages, $\$ 93.75$

This book is an organized overview of nuclear medicine pertinent to clinical practice and has been one of the most popular textbooks for trainees in nuclear medicine and radiology since its first edition in 1995. Each book in "The Requisites" series is written by nationally recognized authorities in their respective subspecialty areas. Each author is challenged to present material in the context of today's practice of radiology in a way that makes it possible to access the most important topic efficiently. This fourth edition closely follows the philosophy and format of the original to provide a concise and updated introduction and review of the nuclear medicine field. Many advances have been made in radiopharmaceuticals and instrumentation since the publication of the third edition in 2006. Standard use of PET/CT has become widely accepted, and SPECT/CT is increasing in clinical use. PET/MR imaging is still in its infancy, but enough data are at hand to predict that this integrated imaging will become important. The integrated methods of SPECT/CT, PET/CT, and PET/MR imaging require higher levels of knowledge of anatomic cross-sectional imaging than ever before, as well as greater knowledge of imaging technology and molecular biology.

This edition contains many new superb images and revised, updated text. The book consists of 2 parts with 17 chapters. Part I, with 5 basic science chapters, deals with important principles of radiopharmaceuticals, has a new chapter on molecular imaging and a rewritten chapter on physics, and has chapters on instrumentation, radiation detection, and hybrid imaging with SPECT and PET. Part II, with 12 clinical chapters, discusses the endocrine, skeletal, hepatobiliary, genitourinary, pulmonary, gastrointestinal, central nervous, and cardiac systems, as well as infection and inflammation, and has extensively revised the oncology chapters and the popular "Pearls, Pitfalls, and Frequently Asked Questions." The clinical chapters continue to follow a logical progression from basic principles of tracer distribution and localization to practical clinical application. Therefore, there is an emphasis on the pharmacokinetics and understanding of disease pathophysiology, leading naturally to the choice of optimal imaging methods and study interpretation.

This book delivers the conceptual, factual, and interpretive information for effective clinical practice of nuclear medicine imaging, as well as for board or certification review. It is a clear, concise text enhanced by optimal illustrations, helpful boxes, tables, suggested reading, and index. I highly recommend this book to medical students, trainees, and practitioners in nuclear medicine and radiology.

\section{E. Edmund Kim}

University of California at Irvine

101 The City Dr. S.

Orange, $C A 92868$

E-mail:edmundek@uci.edu 